\title{
An anesthetic experience of hereditary angioedema type I patient undertook total laparoscopic hysterectomy - A case report -
}

Received August 10, 2021

Revised November 16, 2021

Accepted November 18, 2021

\section{Corresponding author}

Chunghyun Park, M.D., Ph.D.

Department of Anesthesiology and

Pain Medicine, CHA Bundang Medical

Center, CHA University, 59 Yatap-ro,

Bundang-gu, Seongnam 13496,

Korea

Tel: 82-31-780-5157

Fax: 82-31-780-5157

E-mail: anesthpark@chamc.co.kr

\section{Yun-Sic Bang, Jaeho Cho, and Chunghyun Park}

Department of Anesthesiology and Pain Medicine, CHA Bundang Medical Center, CHA University, Seongnam, Korea

Background: Hereditary angioedema (HAE) is an autosomal dominant disorder. The characteristic of HAE is recurrent angioedema episodes due to low C1 esterase inhibitor (C1-INH) level. HAE symptoms, especially those affecting oropharynx or larynx may develop respiratory distress syndrome due to impaired airway, which can be potentially fatal.

Case: We report a clinical case of a 57 year-old female patient, with type I HAE, scheduled for total laparoscopic hysterectomy under general endotracheal anesthesia, which was done successfully without inducing airway edema. Danazol, which increases liver synthesis of C1$\mathrm{INH}$, was administered and fresh frozen plasma (FFP), which contained $\mathrm{C} 1-\mathrm{INH}$, was transfused after induction.

Conclusions: For HAE patients, the greatest concern is that general anesthesia can induces upper airway edema by direct mucosal irritation by the endotracheal tube. The perioperative management should include both prophylactic increase of C1-INH production and on-demand administration of C1-INH or FFP.

Keywords: Anesthesia; Complement C1 inhibitor protein; Danazol; Fresh frozen plasma; Hereditary angioedema; Hysterectomy.
Hereditary angioedema (HAE) is an autosomal dominant genetic disease with a prevalence of 1:50,000. The etiology of HAE includes low level of C1-esterase inhibitor (C1-INH) antigen and/or low activity of C1-INH. The disease is characterized by recurrent, nonpitting edema typically on the upper or lower extremities, genital area, trunk, and face, as well as submucosal edema involving the digestive tract or upper airway tract [1]. The greatest concern of patients with HAE is upper airway edema induced by direct mechanical irritation due to the endotracheal tube during general anesthesia [2]. Laryngeal edema warrants urgent treatment and immediate hospitalization in $37 \%$ of all angioedema epi- sodes [3].

We present a clinical case of a 59-year-old female patient who underwent total laparoscopic hysterectomy under general endotracheal anesthesia, managed successfully.

\section{CASE REPORT}

Institutional Review Board (IRB) waived the requirement of written consent for the publication of this case (no. 202103-060-002 CHA Bundang Medical Center IRB). The patient has also provided prior consent to the publication of procedures and anonymous case details in this report.

This is an Open Access article distributed under the terms of the Creative Commons Attribution Non-Commercial License (http://creativecommons.org/licenses/by-nc/4.0) which permits unrestricted non-commercial use, distribution, and reproduction in any medium, provided the original work is properly cited.

Copyright (C) the Korean Society of Anesthesiologists, 2022 
A 59-year-old female patient (height, $162 \mathrm{~cm}$; weight, 59 $\mathrm{kg}$ ) with type I HAE was scheduled for total laparoscopic hysterectomy. The patient's medical history revealed a diagnosis of type I HAE at the age of 49 years, based on genetic testing. She experienced recurrent episodes of angioedema involving legs or hands once every 2-3 months. However, she had not experienced an edema attack for the past one year, so she had been using $100 \mathrm{mg}$ of danazol once a week for long-term symptom control. Preoperative blood tests revealed normal range of biochemical parameters except for total cholesterol (216 mg/dl, normal range: 0-200 mg/dl). Protein immunoassay revealed a C4 level of $14 \mathrm{mg} / \mathrm{dl}$ (normal range: $10-40 \mathrm{mg} / \mathrm{dl})$. The patient's C1-INH antigen level was $9.70 \mathrm{mg} / \mathrm{dl}$ (normal range: $21.0-39.0 \mathrm{mg} / \mathrm{dl}$ ) and the $\mathrm{Cl}$ INH activity was 33\% (normal range: $70-130 \%$ ). Chest X-ray and electrocardiography findings were normal.

The patient was treated with danazol $200 \mathrm{mg}$ the day before surgery. Before induction, the blood pressure was $135 / 78 \mathrm{mmHg}$, and the pulse rate was 82 beats/min. General anesthesia was induced with intravenous (IV) glycopyrrolate $0.2 \mathrm{mg}$, propofol $100 \mathrm{mg}$, sufentanil $5 \mu \mathrm{g}$ and rocuronium 40 mg. The endotracheal intubation was performed smoothly using a video laryngoscope, and no abnormalities such as edema of the epiglottis or vocal cords were detected. Anesthesia was maintained with an end-tidal concentration of sevoflurane 2-3 volume\%, and intermittent sufentanil with $40 \%$ oxygen. Left radial artery was cannulated. We transfused 3 pints of fresh frozen plasma (FFP) as recommended by the allergy department. At the end of the surgery, video laryngoscopy confirmed the absence of edema in the patient's epiglottis and vocal cord. Further, no edema was detected in the trachea and carina with a fiberoptic bronchoscope. The patient was reversed with glycopyrrolate $0.2 \mathrm{mg}$ mixed with pyridostigmine $10 \mathrm{mg} \mathrm{IV}$, and then extubated after responding to the verbal command. At the end of the operation, an adequate amount of opioid was administered. Spontaneous breathing was restored, followed by extubation with minimal stimulation via the endotracheal tube. The patient did not have any particular complaints and the vital signs were stable. The total fluid intake during the surgery was $1,310 \mathrm{ml}$ including 3 pints of FFP, and the output was $680 \mathrm{ml}$ including blood loss. She stayed at the sub-intensive care unit for 1 day to monitor possible late-onset airway edema after surgery. No special events were detected until discharge, and the patient took danazol as usual and was discharged 4 days after the operation.

The patient visited the outpatient clinic a week after dis- charge and reported no significant event. However, four weeks later, during her second visit, she complained that edema alternated between her left and right feet every 10 days. The patient no longer experienced edema by week 8 of outpatient follow-up.

\section{DISCUSSION}

HAE is divided into three types. Type I HAE appears in about $85 \%$ of HAE patients and is characterized by a low level of C1-INH antigen (less than 35\%) and low inhibitory activity. The functional C1-INH activity in type I HAE is typically $20-35 \%$. Type II HAE is less common and occurs in $\leq$ $15 \%$ of HAE patients; it is associated with a functional defect involving C1-INH. In type II HAE, the C1-INH antigen level is normal or sometimes elevated. In addition, type N HAE is detected in a few cases [1]. Our patient is type I HAE and also had a low C1-INH antigen level of $9.7 \mathrm{mg} / \mathrm{dl}(32 \%)$ and $33 \%$ of low C1-INH activity. The C4 level is simple and inexpensive to test and is used as a screening tool; however, in our patient, the preoperative $\mathrm{C} 4$ level was in the normal range. Rarely, the $\mathrm{C} 4$ levels are normal between angioedema episodes [4]. Low or dysfunctional activity of C1-INH interferes with kallikrein, preventing normal bradykinin synthesis. As a result, bradykinin-mediated vascular permeability is altered [5].

Bork and Barnstedt [6] reported four deaths due to laryngeal edema after tooth extraction. Patients with HAE who require tooth extraction also carry a risk of developing facial or laryngeal edema and prophylactics can be used to reduce the risk of symptoms [7]. Angioedema associated with surgery usually appears in surgical areas, but may occasionally involve unrelated areas. Among 19 reports of angioedema-associated surgery, a case of laryngeal angioedema was detected after laparoscopic surgery and two cases after adenoidectomy. Reintubation was performed to manage a case of laparoscopy-induced laryngeal edema [8]. Despite the absence of a direct correlation between intubation and laryngeal angioedema in HAE patients, laryngeal angioedema has been reported after oral surgery in patients who did not receive prophylactic treatment $[6,7]$. Upper airway stimulation should be avoided as much as possible to ensure safety. In our case, no laryngeal mask airway (LMA) was used because it was a laparoscopic surgery. LMA does not stimulate the vocal cord or trachea, but its large contact area in the oral cavity may lead to oral mucosa stimulation. Fatal airway edema was reported after tooth extraction rather than air- 
way intubation $[6,7]$. Therefore, irritation of the oral mucosa can also cause asphyxia, and in the case of edema, it is considered safe to perform endotracheal intubation.

In general, to prevent postoperative laryngeal edema, an endotracheal tube of adequate size should be selected first. Second, the duration of intubation should be minimized. Third, the cuff pressures should be measured regularly to prevent tracheal stimulation due to high cuff pressure.

Regional anesthesia, such as brachial plexus block or neuraxial block, is considered to be a good alternative for general anesthesia to reduce the possible incidence of airway edema induced by mechanical stimulation due to endotracheal tube.

The two ways to approach HAE patient include preventing attacks with prophylaxis and on-demand treatment of edema episodes. Both are very important in perioperative patients. Patients who have frequent, above-moderate degree of angioedema episodes need prophylactic and on-demand treatment during medical or surgical procedures [9]. Before 2009, antifibrinolytics (e.g., tranexamic acid and $\varepsilon$-aminocaproic acid) and 17beta-hydroxy steroid (danazol) were used as prophylactics to increase the synthesis of C1-INH in liver. A number of agents that have been approved over the past few decades are available for prophylactic use. Between 2008 and 2017, the US Food and Drug Administration approved three plasma-derived C1-INH agents: Cinryze ${ }^{\circledR}$ (2008, Shire ViroPharma, USA), Berinert ${ }^{\circledR}$ (2009, CSL Behring, USA), and Haegarda ${ }^{\circledR}$ (2017, CSL Behring, USA) [10]. Consensus guidelines recommend giving 500-1,500 U of Berinert $^{\circledR}$ as a prophylaxis one hour before the procedure to prevent severe angioedema [11]. In 2018, Takhzyro ${ }^{\circledR}$ (Shire Dyax Corp., USA), a plasma kallikrein inhibitor, was approved [10]. When plasma-derived C1-INH is not available, solvent-treated FFP is recommended $1-2 \mathrm{~h}$ prior to procedure, or administration of danazol is recommended days prior to increased liver synthesis of C1-INH. FFP is more effective in preventing or minimizing angioedema than danazol [11]. In our case, the dose of danazol was raised to only $200 \mathrm{mg}$ a day and FFP was given after induction, because there was no edema in the last one year.

On-demand treatment is needed to reduce the degree of angioedema and pain during HAE attack and induce rapid recovery. Until plasma-derived C1-INH became popular, FFP was the first choice of on-demand treatment and is still the only treatment when C1-INH is not available. A few units of FFP (1-4 U) are effective (96\%) in all types of acute attack including severe subcutaneous and submucosal laryngeal edema. FFP is considered effective in preventing and treating acute angioedema attacks before surgery [12]. However, the time to resolution is slower than in targeted therapies [13]. In addition to Berinert ${ }^{\circledR}$, Ruconest ${ }^{\circledR}$ (Pharming, Bridgewater, USA), which is a recombinant C1-INH, can also be used as a prophylactic. Firazyr ${ }^{\circledR}$ (bradykinin antagonist, Shire, USA) and Kalbitor ${ }^{\circledR}$ (Shire Dyax Corp.) are also used as treatments [10]. In HAE patients over 12 years of age, 20 $\mathrm{IU} / \mathrm{kg}$ of Berinert ${ }^{\circledR}$ is used for treatment [14].

We transfused 3 pints of FFP immediately before surgery in this patient and extubated safely. In Korea, Berinert ${ }^{\circledR}$ was approved by the Korean Ministry of Drugs and Food Safety in 2011, and on April 1, 2021, CSL Korea signed a contract to import Berinert ${ }^{\circledR}$, although it has yet to be imported. Therefore, the best option was to use danazol as a prophylactic treatment and FFP as a prophylactic and on-demand treatment under the circumstances prevailing in South Korea.

In HAE, an edema follows a predictable course. In many cases, it involves prodromal signs such as a tingling sensation or a non-itching serpiginous rash. Swelling classically progresses slowly but continues for the first 24 hours, followed by a gradual decline over the subsequent 48 to $72 \mathrm{~h}$. According to one report, laryngeal angioedema is symp-

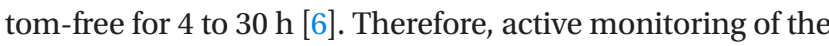
patient should be performed at least one day after the surgery. Fortunately, there was no airway edema in our patient following extubation after the surgery. However, the patient was monitored in the sub-intensive care unit due to the risk of angioedema up to $12-24 \mathrm{~h}$ after surgery.

Several weeks after discharge, the patient present at the outpatient clinic with complaints of recurring foot edema, despite the continued use of danazol.

Successful management of HAE requires close and appropriate teamwork between the departments of allergy and immunology, surgery, anesthesiology and critical care medicine for accurate diagnosis, prevention, and treatment.

Histamine-mediated angioedema (HMA) must be distinguished from HAE, which exhibits a similar clinical pattern. HMA is often accompanied by urticaria and pruritus, lasts for 24-48 h, and responds to administration of antihistamines and corticosteroids [15]. HAE does not show histamine-mediated wheals or bronchoconstriction; therefore, steroids or antihistamines are not very effective in treating HAE.

The standard treatment for HAE is prophylactic Cl-INH before surgery and on-demand treatment of recombinant C1-INH for symptom management, and close observation 
for at least $24 \mathrm{~h}$ after surgery for delayed angioedema. However, if C1-INH is not available, prophylactic danazol and FFP should be administered before surgery, as in our case, followed by close observation for $24 \mathrm{~h}$. It is also recommended to use FFP for therapeutic purposes even if symptoms appear. In conclusion, edema prophylaxis and on-demand treatment are important since airway edema can be induced by surgical stress. In addition, careful observation after surgery is necessary to rapidly detect delayed manifestations of edema.

\section{FUNDING}

None.

\section{CONFLICTS OF INTEREST}

No potential conflict of interest relevant to this article was reported.

\section{DATA AVAILABILITY STATEMENT}

None.

\section{AUTHOR CONTRIBUTIONS}

Project administration: Chunghyun Park. Writing - original draft: Yun-Sic Bang, Jaeho Cho, Chunghyun Park. Writing - review \& editing: Yun-Sic Bang. Investigation: Yun-Sic Bang. Supervision: Chunghyun Park.

\section{ORCID}

Yun-Sic Bang, https://orcid.org/0000-0002-0930-4313

Jaeho Cho, https://orcid.org/0000-0002-5181-6937

Chunghyun Park, https://orcid.org/0000-0003-1916-6644

\section{REFERENCES}

1. Zuraw BL. Clinical practice: hereditary angioedema. N Engl J Med 2008; 359: 1027-36.

2. Farkas H. Management of upper airway edema caused by hereditary angioedema. Allergy Asthma Clin Immunol 2010; 6: 19.

3. Bork K, Barnstedt SE. Treatment of 193 episodes of laryngeal edema with $\mathrm{C} 1$ inhibitor concentrate in patients with hereditary angioedema. Arch Intern Med 2001; 161: 714-8.
4. Zuraw BL, Sugimoto S, Curd JG. The value of rocket immunoelectrophoresis for $\mathrm{C} 4$ activation in the evaluation of patients with angioedema or C1-inhibitor deficiency. J Allergy Clin Immunol 1986; 78: 1115-20.

5. Davis AE 3rd. Mechanism of angioedema in first complement component inhibitor deficiency. Immunol Allergy Clin North Am 2006; 26: 633-51.

6. Bork K, Barnstedt SE. Laryngeal edema and death from asphyxiation after tooth extraction in four patients with hereditary angioedema. J Am Dent Assoc 2003; 134: 1088-94.

7. Bork K, Hardt J, Staubach-Renz P, Witzke G. Risk of laryngeal edema and facial swellings after tooth extraction in patients with hereditary angioedema with and without prophylaxis with C1 inhibitor concentrate: a retrospective study. Oral Surg Oral Med Oral Pathol Oral Radiol Endod 2011; 112: 58-64.

8. Aygören-Pürsün E, Martinez Saguer I, Kreuz W, Klingebiel T, Schwabe D. Risk of angioedema following invasive or surgical procedures in HAE type I and II--the natural history. Allergy 2013; 68: 1034-9.

9. Bowen T, Cicardi M, Farkas H, Bork K, Longhurst HJ, Zuraw B, et al. 2010 International consensus algorithm for the diagnosis, therapy and management of hereditary angioedema. Allergy Asthma Clin Immunol 2010; 6: 24.

10. Tanaka KA, Mondal S, Morita Y, Williams B, Strauss ER, Cicardi M. Perioperative management of patients with hereditary angioedema with special considerations for cardiopulmonary bypass. Anesth Analg 2020; 131: 155-69.

11. Bowen T, Cicardi M, Bork K, Zuraw B, Frank M, Ritchie B, et al. Hereditary angiodema: a current state-of-the-art review, VII: Canadian Hungarian 2007 international consensus algorithm for the diagnosis, therapy, and management of hereditary angioedema. Ann Allergy Asthma Immunol 2008; 100(1 Suppl 2): S30-40.

12. Prematta M, Gibbs JG, Pratt EL, Stoughton TR, Craig TJ. Fresh frozen plasma for the treatment of hereditary angioedema. Ann Allergy Asthma Immunol 2007; 98: 383-8.

13. Wentzel N, Panieri A, Ayazi M, Ntshalintshali SD, Pourpak Z, Hawarden D, et al. Fresh frozen plasma for on-demand hereditary angioedema treatment in South Africa and Iran. World Allergy Organ J 2019; 12: 100049.

14. Craig TJ, Bewtra AK, Bahna SL, Hurewitz D, Schneider LC, Levy RJ, et al. C1 esterase inhibitor concentrate in 1085 hereditary angioedema attacks--final results of the I.M.P.A.C.T.2 study. Allergy 2011; 66: 1604-11.

15. Busse PJ, Smith T. Histaminergic angioedema. Immunol Allergy Clin North Am 2017; 37: 467-81. 